\title{
Impact of poor fragmentation on cave management
}

\author{
S.N. Ngidi Palabora Mining Company, South Africa \\ D.D. Pretorius Palabora Mining Company, South Africa
}

\begin{abstract}
Palabora Mining Company operates a low grade underground copper mine situated in the Limpopo Province of South Africa about $560 \mathrm{~km}$ north east of Johannesburg. The mine is managed by the Rio Tinto Group and has been in operation since 1965.

Full production underground was first reached in May 2005 after a successful transition from an open pit to a block cave mine. The cave is subdivided into four sectors comprising of 19 operational crosscuts. Caving was initiated in the weaker central sector, where the majority of discontinuities are located. This central sector is directly under the open pit bottom which is currently filled with over 150 million tonnes of waste material from the 2004 pit wall failure.

The main constraint during the ramp up stage has been the poor fragmentation on the western side of the cave. The western section of the footprint, Sector 4, has the youngest drawpoints and the highest draw columns. About $36 \%$ of the ore reserves have to be extracted from Sector 4 which has a much coarser fragmentation compared to the rest of the cave. Estimation of practical production rates within acceptable limits had to be reviewed in order to enhance long-term production scheduling. Cave management and operational strategies had to be properly aligned with these challenges in order to achieve maximum ore reserve extraction.
\end{abstract}

\section{Introduction}

Undercutting for the Palabora Cave was initiated from the centre of the footprint taking advantage of the two major fault structures and the presence of dolerite dykes and then advancing to the west and the east. The western sector of the cave is dominated by blocky banded carbonatite whilst the eastern sector consists mainly of transgressive carbonatite.

The cave is divided into four sectors of five crosscuts each with Sector 1 on the east and Sector 4 on the western side of the cave. A total of $36 \%$ of the ore reserves has to be extracted from Sector 4 - which has a much coarser fragmentation compared to the other sectors. This sector lies the furthest to the west from the cave initiation point and hence the last sector to come to full production. Sectors 2 and 3 have a much finer fragmentation and a $41 \%$ combined share of the reserves. These sectors have the majority of their crosscuts located directly below the open pit bottom, hence the shorter draw columns.

Detailed in this paper are the challenges and impacts posed by fragmentation on cave management both on the operational and technical level. Predicted fragmentation during feasibility was then compared to actual data and production plans and operational processes altered accordingly.

\section{Background}

The Palabora block cave is serviced by two shafts with a herringbone layout consisting of 166 drawbells. These drawpoints make up 19 crosscuts from which ore is loaded and tipped into four $750 \mathrm{t} / \mathrm{hr}$ jaw crushers. The footprint covers approximately $119,000 \mathrm{~m}^{2}$ with drawpoints ranging between 8 and 21 per crosscut.

Tonnage is currently drawn from 314 active drawpoints with an average availability of about $65 \%$. 


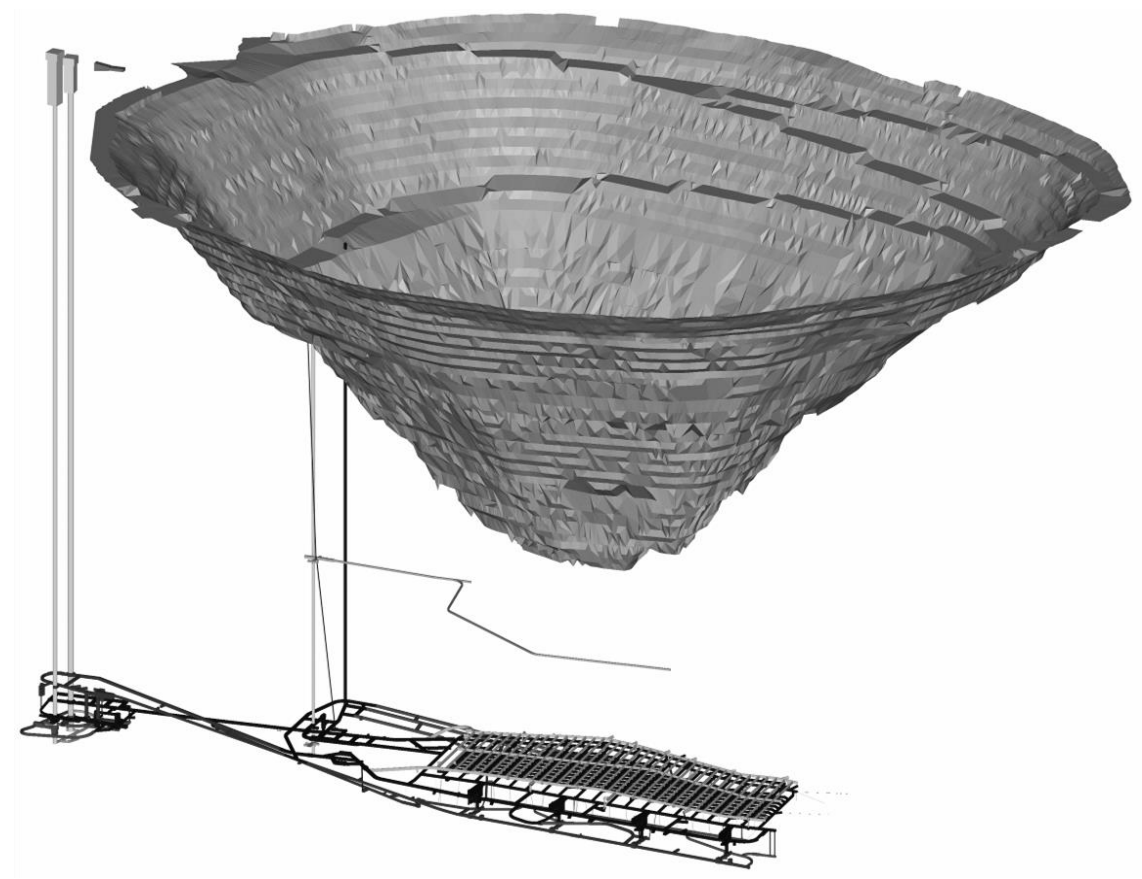

Figure 1 Palabora block cave mine layout (east-west)

The current production level is located at a depth of about 1,200 $\mathrm{m}$ below surface. Column heights range between $397 \mathrm{~m}$ directly below the open pit floor to $760 \mathrm{~m}$ for the furthest columns in the western sector. The Palabora underground mining operation currently supplies an average of 33,000 tonnes of copper ore to the company's concentrating, smelting and refinery plants on site, for the production of copper rod and cathode.

\section{Rock mass properties}

The average uniaxial strength of the carbonatites is about $120 \mathrm{MPa}$, with a variation between $90 \mathrm{MPa}$ and $160 \mathrm{MPa}$ in values depending on mineralogy. Dolerite is a strong brittle rock with a uniaxial strength of $320 \mathrm{MPa}$. Adjacent to the major faults, dolerite is locally weathered with a marked reduction in strength to approximately $80 \mathrm{MPa}$.

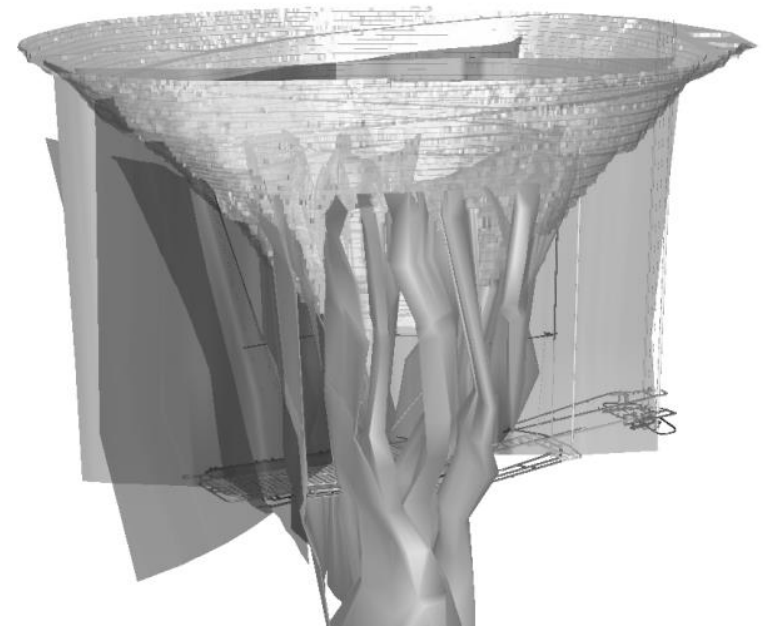

Figure 2 3D model of the dolerite dykes and fault zones (west-east)

The structure of the carbonatites is predominantly subvertical jointing. These joints are planar and persistent in nature and can be open or infilled with weak material. There are three steeply dipping sets, striking approximately $010^{\circ}$ (dip direction $290^{\circ}$ ), $310^{\circ}$ (dip direction $040^{\circ}$ ) and $050^{\circ}$ (dip direction $140^{\circ}$ ). The flat 
lying joints have a different morphology from the vertical sets: wavy, rough and of limited continuity. There are two sets oriented approximately $20 / 160^{\circ}$ and $45 / 350^{\circ}$. For the purposes of the fragmentation analysis, the rock fabric was idealised to three major sets.

The fracturing of dolerite is closely spaced and blocky. Within the cave area, the fracturing in dolerite was established to be zoned by proximity to the major faults.

\section{$4 \quad$ Fragmentation}

During the project feasibility study a fragmentation prediction program (Block Cave Fragmentation - BCF) was specifically developed by D.H. Laubscher and G.L. Esterhuizen to generate rock size distribution using, as input data, the cave face orientation (dip and dip direction), stress data, mining rock mass rating (MRMR), joint set orientations and joint set spacing (Esterhuizen, 1994). The program also caters for secondary fragmentation, which is the breakage of primary blocks into smaller fragments, through repeated splitting, corner rounding and splitting when temporary arches fail. Results from the BCF model were calibrated using actual data from Premier and the Codelco caves in Chile. It is known that BCF predictions are generally conservative.

Estimates using the BCF program indicated that in the first year of production over $75 \%$ of the rock would be greater than $2 \mathrm{~m}^{3}$ and contribute up to a maximum of $80 \%$ of the total tonnage mined. To the contrary the actual data indicates that the oversize material contributes about $30 \%$ to the total tonnes mined.

Total hang-up predictions were, however, understated by the program in relation to the oversize rocks and the number of high hang-ups was overstated.

\section{Oversize rocks}

Oversize rocks resulting from the caving process are treated by either blasting or physical breaking using a mobile or fixed rock breaker. In Palabora all the rocks larger than $2 \mathrm{~m}^{3}$ were blasted in the drawpoints using robust rigs and non-explosives technology. In 2007 this secondary breakage activity was augmented by the introduction of the mobile rock breakers. These are load-haul-dump (LHD) units fitted with a hydraulic hammer. Four mobile rock breakers are currently in operation.

Over 300 oversize rocks are broken per day. Contrary to the feasibility predictions, rocks broken per day have been on the increase for the past nine years and do not seem to have reached a steady state except for Sector 1 . This is, however, not in line with maturity and comminution rules. The increase observed in Sectors 3 and 4 after 2007 is believed to be due to the introduction of mobile rock breakers and the tendency to break rocks smaller than $2 \mathrm{~m}^{3}$. This is confirmed by the decrease in the number of crusher blockages observed since 2007.

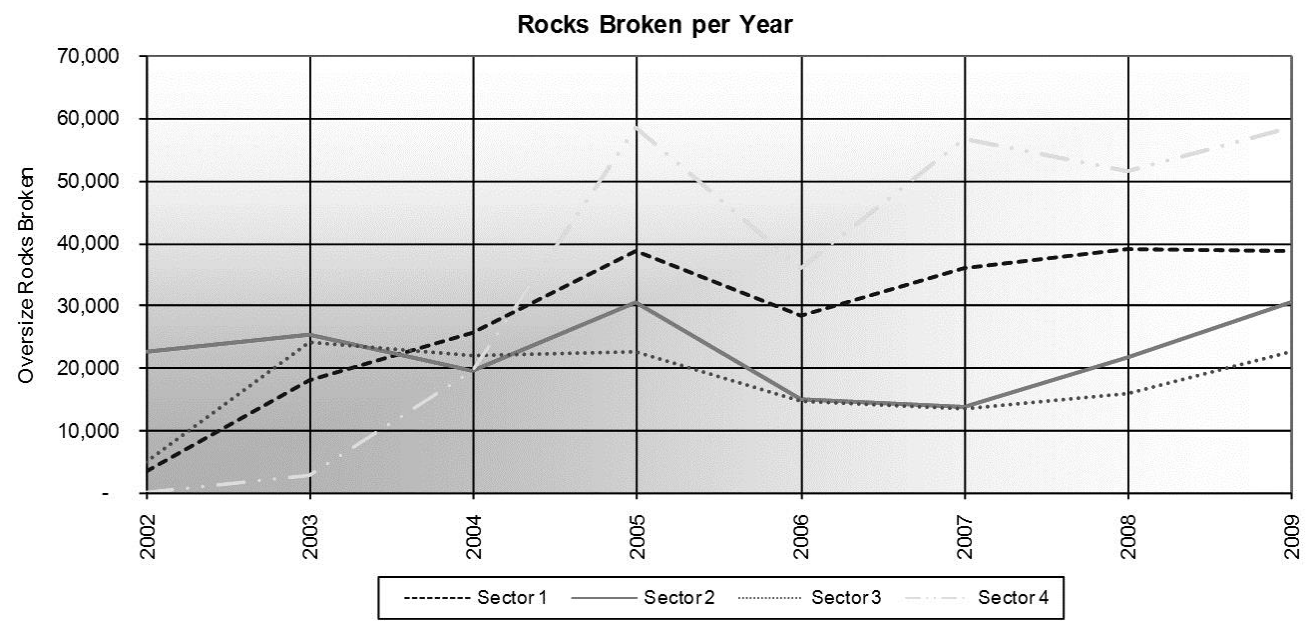

Figure 3 Rocks broken per sector 
The trend for total rocks broken has been stable between 2005 and 2007. All the robust rigs previously used for breaking rocks have since been replaced by mobile rock breakers. In order to minimise operational delays (due to rock breaking) and improve loading time, these machines operate in the same vicinity where LHDs are loading. Prior to this change LHDs would be withdrawn from a crosscut to allow for slow moving rigs to carry out the rock breaking process.

Sector 4 is the highest contributor for the total number of rocks broken. Of the total number of rocks broken per day, Sector 4 contributes just over $40 \%$ and seems to have stabilised at that level. Contribution from Sector 1 is declining which is in line with the geology and maturity rules. Contribution from Sector 2 where the cave was initiated dropped drastically between 2002 and 2003 and is currently about $20 \%$ of the total number of rocks broken annually.

Feasibility predictions indicated that for the first three years (2000-2002) oversize would contribute up to $80 \%$ of the total tonnes mined and would eventually drop to about $40 \%$ in 2009 and then rise to $80 \%$ in 2021. Actual records indicate that there was an increase to about $60 \%$ during production ramp-up followed by a sharp decline to an average of about $20 \%$. Average oversize tonnage contribution for 2009 was $28 \%$.

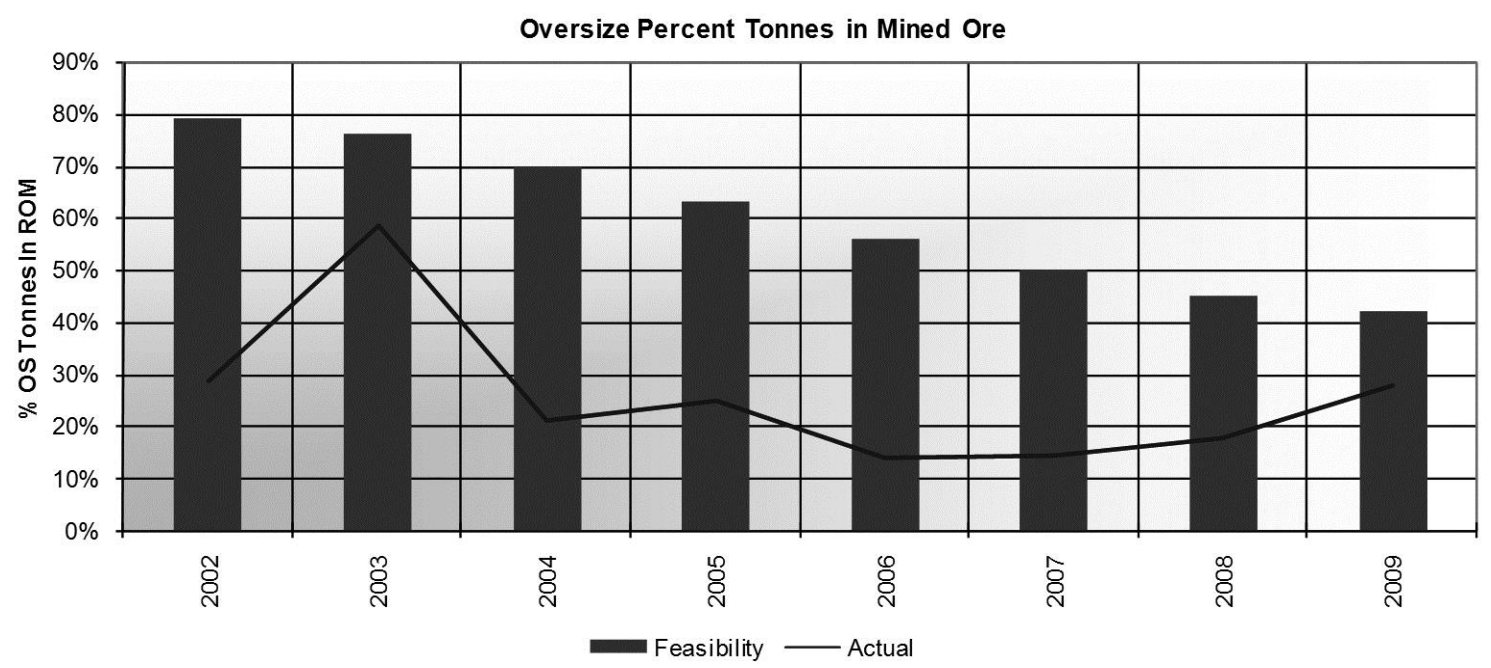

Figure 4 Percentage oversize tonnes in run of mine

Figure 5 below depicts a steady tonnage build-up by sector reaching full production in 2005. Crosscut construction in Sector 4 was completed in 2004 hence the lower tonnage contribution prior to 2005. There was a slight decline in 2008 due to roadway construction and for the first 10 months of 2009 this sector is ahead having contributed about $30 \%$. The life of mine target contribution for this sector is $36 \%$.

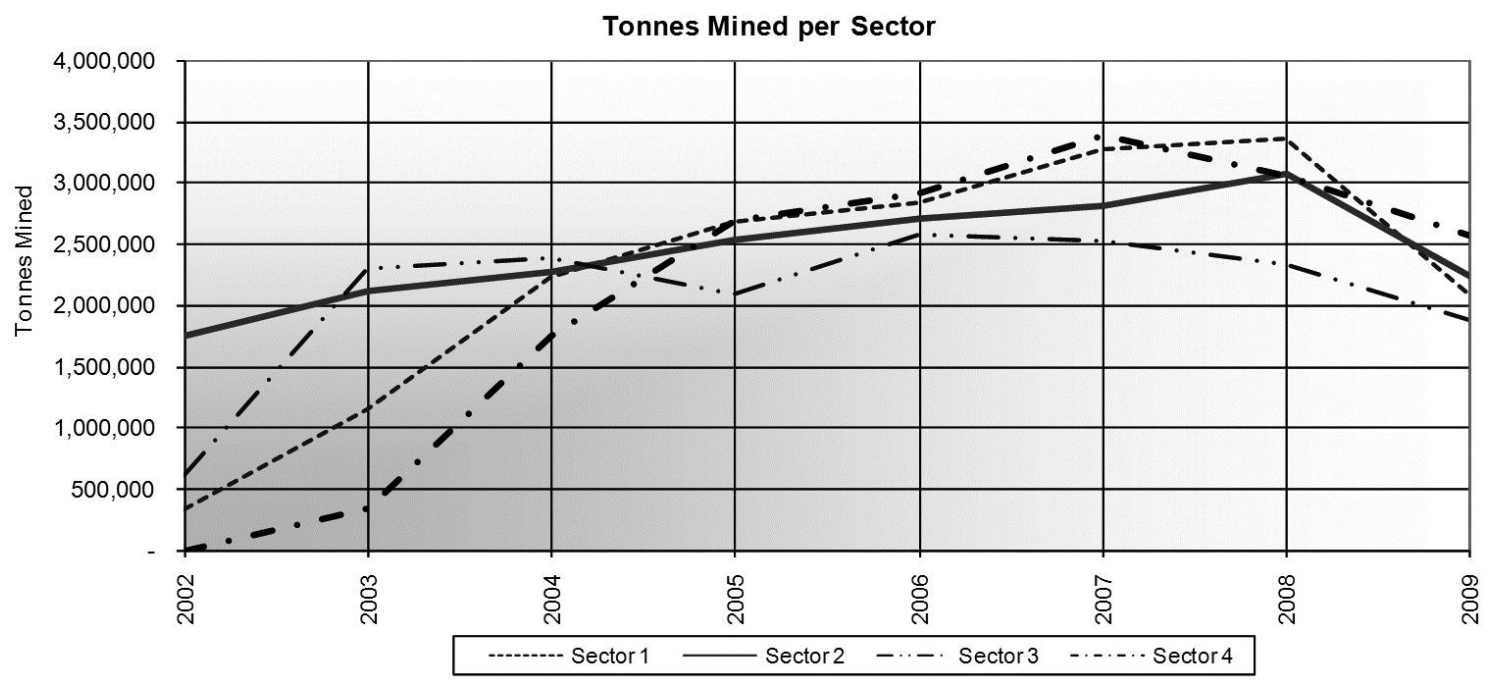

Figure 5 Tonnes mined per sector 


\section{$6 \quad$ Hang-up treatment}

Hang-ups resulting from the caving process are treated by drilling and blasting or simply placing a concussion bag below or between oversize rocks wedged in the drawpoint. The secondary breaking unit section (SBU) has a fleet of four medium reach rigs for drilling or placement of concussion bags.

Hang-ups are stabilised with water cannons pumping up to 12,000 litres at high pressure to wash out all the loose fines between oversize blocks. Over $40 \%$ of the hang-ups come down during this process, and the remainder would then require drilling and blasting. High hang-ups are stabilised and treated with concussion bags until safe drilling with medium reach rigs is achievable.

The number of hang-ups predicted (during feasibility studies) to occur beyond the reach of the medium reach rigs prompted the purchase of the custom designed high reach rig for hang-ups occurring up to $16 \mathrm{~m}$ above the drawpoint floor (Moss et al., 2004). Two rigs of this nature were planned and only one was purchased, although never used.

Hang-ups above $9 \mathrm{~m}$ from the floor (high hang-ups) were projected to reach 24 per day in 2002 and decrease sharply to 16 events per day in 2004 and eventually zero beyond 2009.

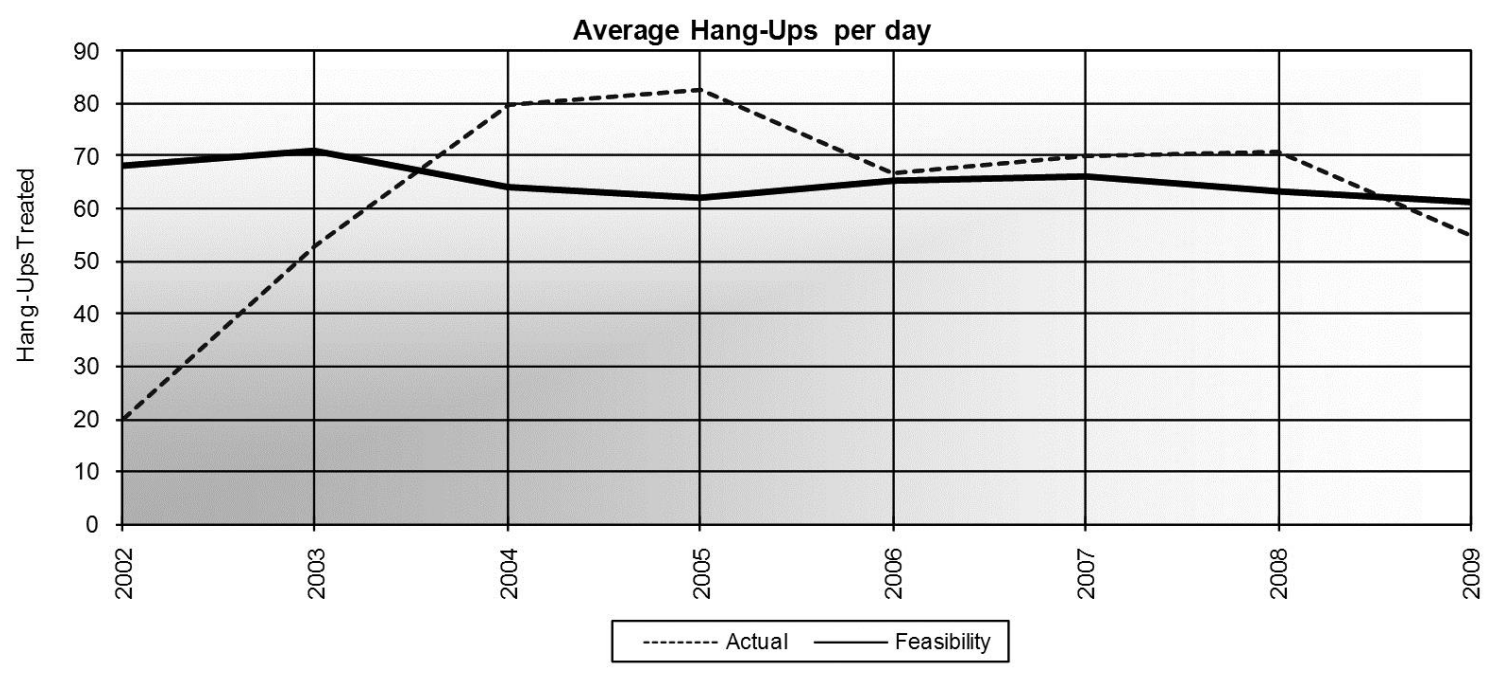

\section{Figure 6 Average hang-ups per day}

An average of 70 hang-ups is treated by the production crews each day. Hang-ups treated per day increased steadily from a meagre average of about 20 per day in 2002 to 84 per day in 2005 . Between 2005 and 2009 there has been an overall decrease in hang-ups treated which is indicative of a maturing cave. Feasibility predictions indicated a lower number of events peaking in 2003 to about 46 per day and later stabilising at 70 events per day and culminating between 60 and 70 for the latter years. Included in the events are high hang-ups requiring treatment with the high reach rig and reaching highs of about 24 events per day in 2002, declining to 16 in 2004 and zero beyond 2009. There is no historical split in the records between high and low hang-ups and hence they cannot be compared. However, it is known that the high reach rig has never been utilised for any rock breaking activities in the history of the project.

Figure 7 indicates that there was a sharp decline in hang-ups treated in Sector 2 between 2003 and 2006. Total hang-ups treated in Sector 2 continued to drop to current values of less than 4,000 per annum. This is in line with the geology and tonnage plan for this area. Sector 4 has the highest number of hang-ups treated per period and currently contributes about $40 \%$ to the total hang-ups treated per day. Sector 1 contributes about $30 \%$ and there are signs of a decline indicative of maturity for the drawpoints in this area. 


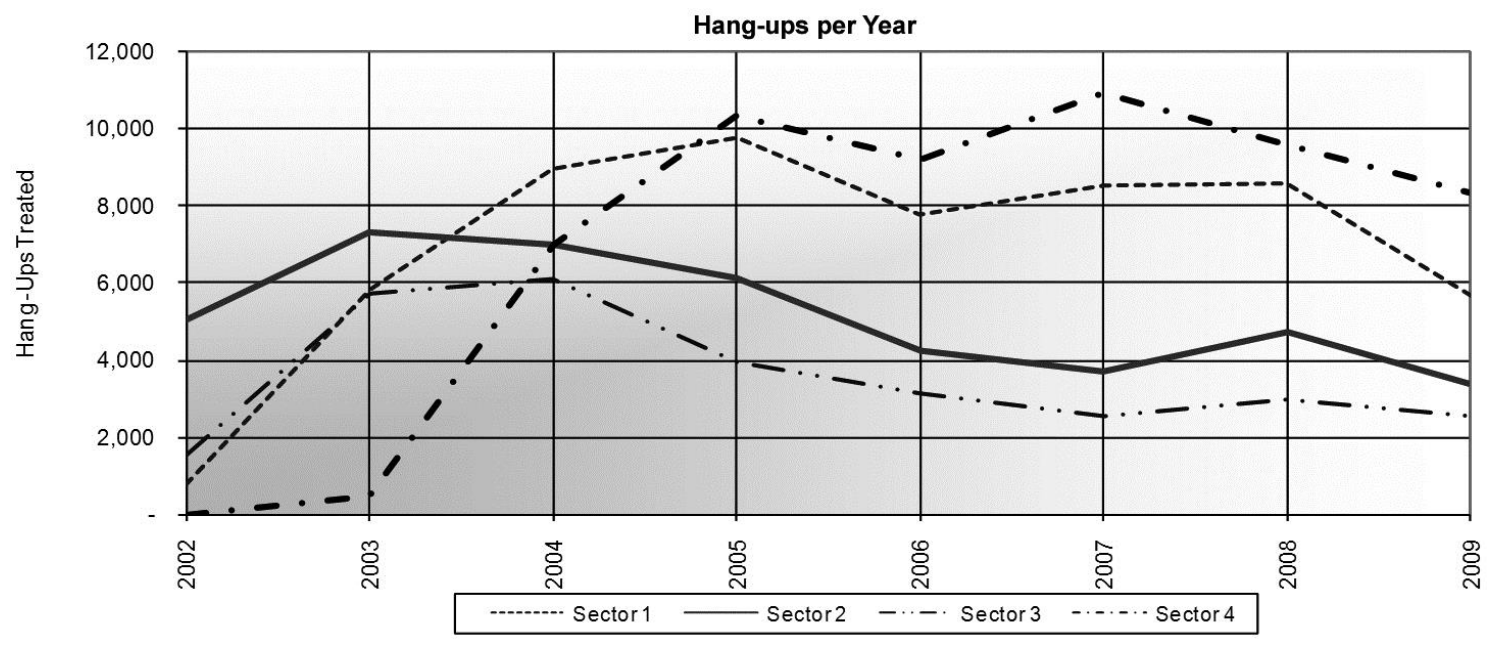

\section{Figure 7 Hang-ups treated per sector}

Tonnes liberated per hang-up treated (yield) as depicted in Figure 8 increased steadily between 2004 and 2007, thereafter declining to an average of about 435 for 2009. Tonnes mined between hang-ups (yield), remains high for Sectors 2 and 3 in line with the good fragmentation as a result of the geology in this zone. Current yield for Sectors 1 and 4 is below 400 tonnes per hang-up. These sectors are characterised by poor fragmentation.

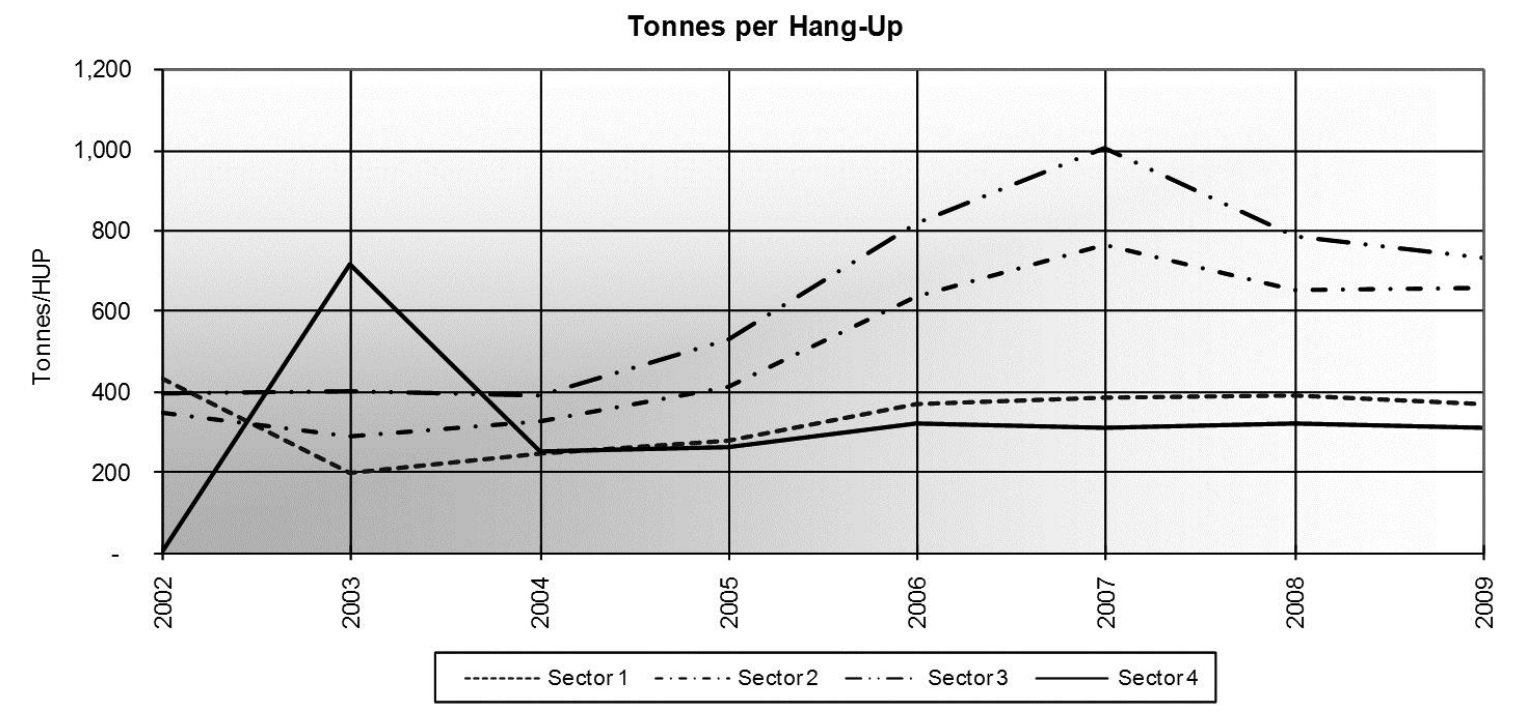

\section{Figure 8 Tonnage yield per sector}

\section{Cave management strategy}

Draw control or cave management is defined as the practice of controlling the tonnages drawn from individual drawpoints with the objective of minimising overall dilution, maintaining the planned ore grade sent to the plant and ensuring maximum ore recovery. Good draw control could also aid in avoiding damaging load concentration on the extraction horizon, optimising fragmentation, avoiding air blasts, and mud rushes (Laubscher, 1994).

Palabora's draw control strategy is aimed at delaying dilution entry and optimising draw from the poorly fragmented western side of the footprint. Originally, the mine employed a daily draw order system developed by Robin Kear. This was a Linux based system with limited capabilities.

This was replaced in 2004 with Gemcom's Personal Computer Block Cave (PCBC) and Cave Management System (CMS) systems. Even draw is applied ensuring maximum ore extraction from the reserves. 
Annual and monthly plans are generated using PCBC as shown in Figure 9 below. Monthly plans generated in PCBC are fed into CMS from which relevant plans on a shift basis are generated. Actual data captured through the dispatch system is transferred back to CMS for reconciliation and database update.

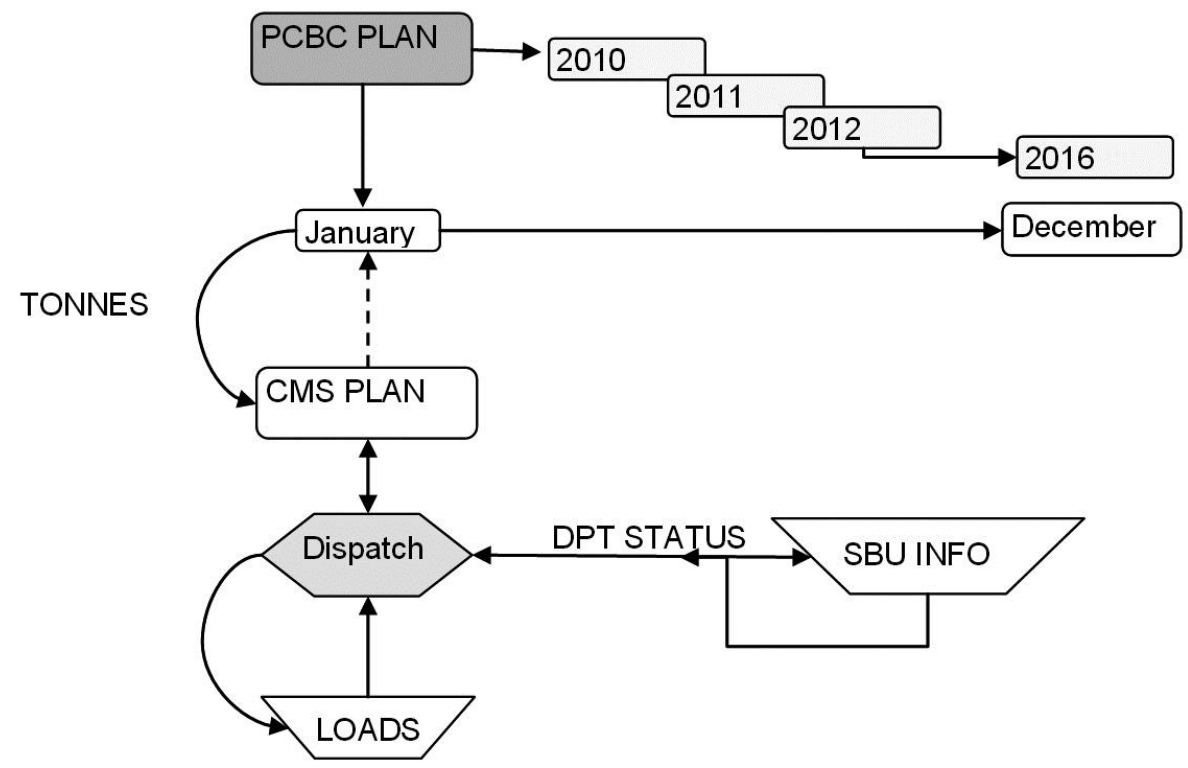

Figure 9 Production scheduling - process flow

Palabora had been using a daily draw order in the initial start up and up until early 2006. With full production realised in 2005, the next step was the implementation and enforcement of relevant draw control rules. During this process it was evident to the scheduling engineers that the daily draw order was inadequate for Palabora's mining conditions. Scheduling engineers trialled and initiated a shift specific draw order system.

The main driver for this was the need for improved draw compliance and the opportunity to be more responsive to the changing drawpoint status (low yield and availability). With the high number of hung up drawpoints and their low yield, the daily draw order was found to be rigid and incompatible with these dynamic conditions and hence the implementation of individual shift production schedules (Diering, 2004).

Palabora uses the dispatch system, a product of Modular Mining Systems Africa (MMSA), to send and receive loading and cave status information. This is a live system through which all loading and rock breaking activities are captured, monitored and reported. The production fleet controller (dispatcher) can send and receive live information through the leaky feeder network to and from the equipment in the field. Additional functionalities such as 'draw order completion' warnings were added to the system to improve operator draw order compliance. The entire secondary breaking unit fleet is also equipped with the dispatch system to give operators access to real-time cave status. This information is then used by supervisors to prioritise drawpoints and crosscuts that require immediate secondary breaking attention.

The prioritisation of Sector 4 which has the highest draw columns shows a marked improvement in the average availability of drawpoints and mucking rates as per generated draw order (draw compliance) as shown in Figure 10. 


\section{Sector 4 Performance Trends}

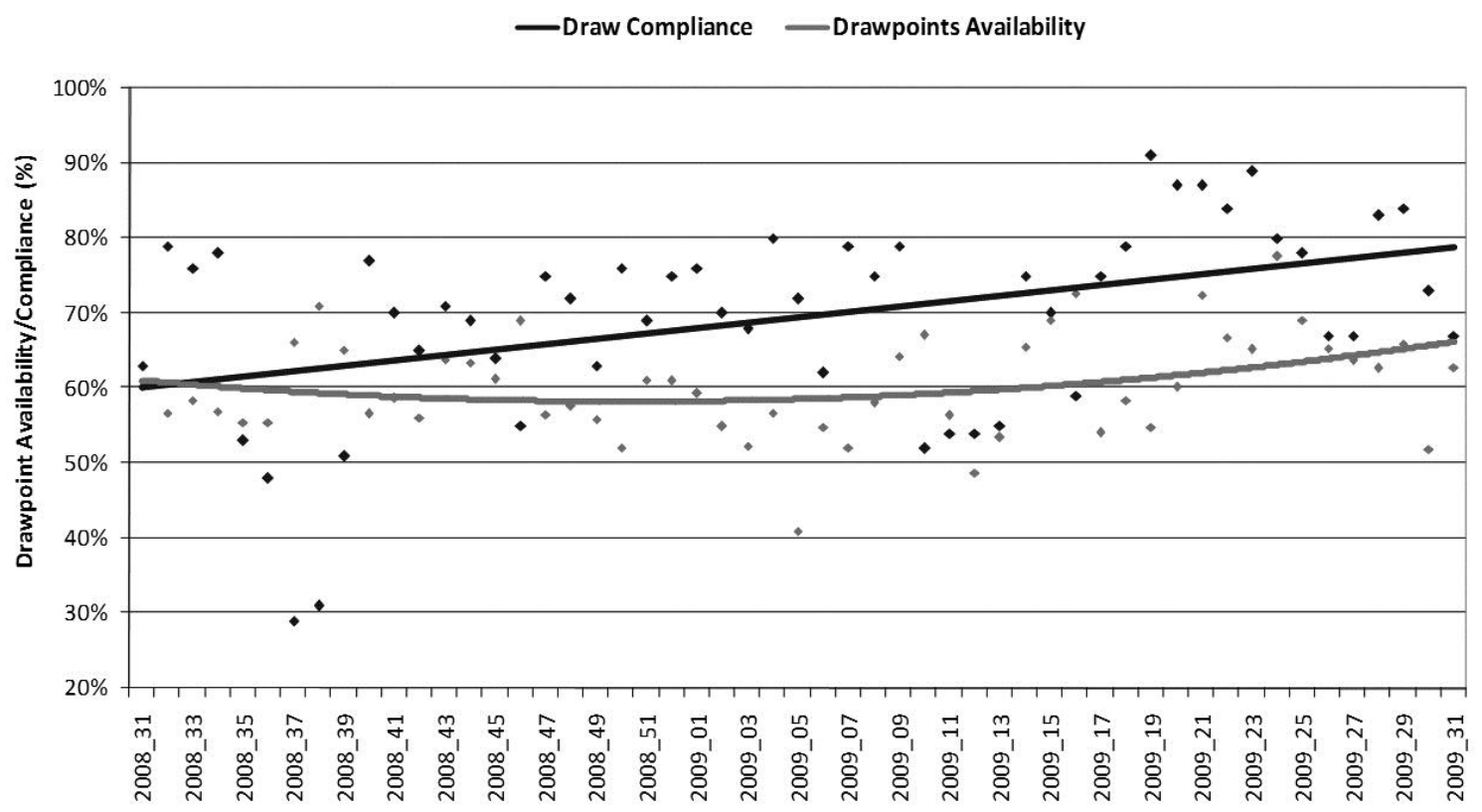

Figure 10 Sector 4 compliance performance

\section{$8 \quad$ Production rate curves}

In 2007 actual drawpoint tonnage and height of draw data accumulated since 2002 was used to establish new production rate curves for use in PCBC. This method replaces the use of programs similar to the BCF used during feasibility. The main objective is to estimate drawpoint tonnage capacity for use in long-term production projections.

The drawpoints were grouped into four sectors and each sectorial rate curve computed. Figure 11 depicts the average production rate per day curve enhanced with the standard deviation to generate the production rate curve to be used as an input in PCBC.

\section{Sector4}

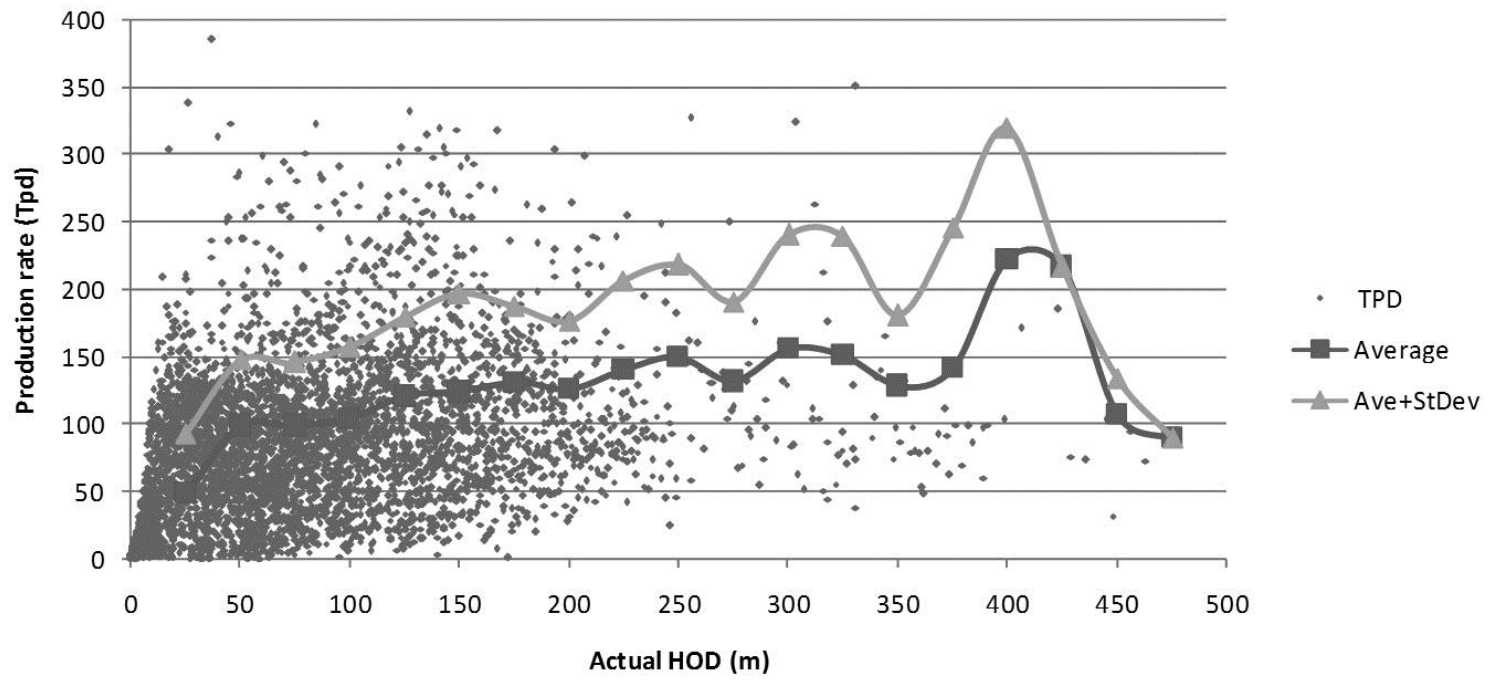

Figure 11 Tonnes per day versus height of draw 
A similar exercise was conducted for all the remaining sectors and the results are summarised in Table 1 . Although this is merely a statistical analysis of historical performance, it is believed that it will enhance the production scheduling process and improve reserve estimations.

\section{Table 1 Historical production performance per sector}

\begin{tabular}{|c|c|c|c|c|c|c|c|c|c|}
\hline $\begin{array}{c}\text { HOD } \\
m\end{array}$ & $\begin{array}{c}\% \text { Extract } \\
\%\end{array}$ & $\begin{array}{l}\text { Sector } 1 \\
\text { Tpd }\end{array}$ & $\begin{array}{c}\text { Sector } 2 \\
\text { Tpd }\end{array}$ & $\begin{array}{c}\text { Sector3 } \\
\text { Tpd }\end{array}$ & $\begin{array}{c}\text { Sector4 } \\
\text { Tpd }\end{array}$ & $\begin{array}{c}\text { Sector1 } \\
\text { Ton/m2-day }\end{array}$ & $\begin{array}{c}\text { Sector } 2 \\
\text { Ton/m2-day }\end{array}$ & $\begin{array}{c}\text { Sector3 } \\
\text { Ton/m2-day }\end{array}$ & $\begin{array}{c}\text { Sector } 4 \\
\text { Ton/m2-day }\end{array}$ \\
\hline 25 & $5 \%$ & 80 & 80 & 95 & 90 & 0.28 & 0.28 & 0.33 & 0.31 \\
\hline 50 & $10 \%$ & 120 & 90 & 130 & 145 & 0.42 & 0.31 & 0.45 & 0.50 \\
\hline 75 & $15 \%$ & 140 & 105 & 135 & 150 & 0.48 & 0.36 & 0.47 & 0.52 \\
\hline 100 & $19 \%$ & 150 & 110 & 145 & 165 & 0.52 & 0.38 & 0.50 & 0.57 \\
\hline 125 & $24 \%$ & 160 & 115 & 165 & 180 & 0.55 & 0.40 & 0.57 & 0.62 \\
\hline 150 & $29 \%$ & 175 & 125 & 175 & 200 & 0.61 & 0.43 & 0.61 & 0.69 \\
\hline 175 & $34 \%$ & 180 & 125 & 175 & 200 & 0.62 & 0.43 & 0.61 & 0.69 \\
\hline 200 & $39 \%$ & 185 & 125 & 175 & 200 & 0.64 & 0.43 & 0.61 & 0.69 \\
\hline 400 & $78 \%$ & 200 & 125 & 175 & 200 & 0.69 & 0.43 & 0.61 & 0.69 \\
\hline 425 & $82 \%$ & 200 & 125 & 175 & 200 & 0.69 & 0.43 & 0.61 & 0.69 \\
\hline 450 & $87 \%$ & 200 & 125 & 175 & 200 & 0.69 & 0.43 & 0.61 & 0.69 \\
\hline 475 & $92 \%$ & 200 & 125 & 175 & 200 & 0.69 & 0.43 & 0.61 & 0.69 \\
\hline
\end{tabular}

\section{Conclusion}

The high yield centre portion of the footprint provided a stable source of tonnes during the ramp-up stage, whilst tonnes mined from Sector 4 were minimal due to the mining challenges associated with poor fragmentation.

Treatment of hang-ups and oversize material prevailing in Sector 4 requires extended hours of rock breaking activities significantly reducing loading time. This results in drawpoints not meeting their daily and monthly planned tonnes. The introduction of mobile rock breakers and the use of concussion bags have significantly increased time available for loading, improving draw compliance and reserve recovery in Sector 4. Poor fragmentation has a huge potential for significantly compromising cave management protocols and reducing mineable reserves. Had these activities not been optimised to improve draw, a significant fraction of the reserves would have been written off due to early dilution.

Oversize prediction using the BCF model was overstated by a significant margin whilst hang-up projections were slightly understated. The exaggeration of the oversize contribution towards total production resulted in an overstated frequency of high hang-ups that can only be addressed with the high reach rig.

In caves where there is a vast differences in geology and column heights, using average estimates could lead to significant reserve losses and hence the need for zone specific production scheduling input parameters. The use of sector specific production rate curves improves reserves estimation and production projections by reducing the errors associated with averages.

\section{Acknowledgements}

The authors wish to gratefully acknowledge Palabora Mining Company for permission to use and publish the information.

\section{References}

Laubscher, D.H. (1994) Cave mining - the state of the art, The Journal of the South African Institute of Mining and Metallurgy, Vol. 94, No. 10, pp. 279-293.

Moss, A., Russell, F. and Jones, C. (2004) Caving and fragmentation at Palabora: Prediction to Production, in Proceedings MassMin 2004, A. Karzulovic and M. Alvaro (eds), 22-25 August, Santiago, Chile, Instituto de Ingenieros de Chile, Santiago, pp. 585-590.

Diering, D., (2004) Combining long term scheduling and daily draw control for block cave mines, in Proceedings MassMin 2004, A. Karzulovic and M. Alvaro (eds), 22-25 August, Santiago, Chile, Instituto de Ingenieros de Chile, Santiago, pp. 486-490 
Esterhuizen, G.S. (1994) A program to predict block cave fragmentation, Technical reference and users guide, Ver 2.1. 